\title{
HAMILTONICITY AND FORBIDDEN SUBGRAPHS IN 4-CONNECTED GRAPHS
}

\author{
FLORIAN PFENDER
}

\begin{abstract}
Let $T$ be the line graph of the unique tree $F$ on 8 vertices with degree sequence $(3,3,3,1,1,1,1,1)$, i.e. $T$ is a chain of three triangles. We show that every 4 -connected $\left\{T, K_{1,3}\right\}$-free graph has a hamiltonian cycle.
\end{abstract}

\section{INTRODUCTION}

For a family $\mathcal{F}$ of connected graphs, a graph is called $\mathcal{F}$-free if it contains no induced copies of any member of $\mathcal{F}$.

A well-known conjecture of Matthews and Sumner [9] states that all 4-connected claw-free (i.e. $\mathcal{F}=\left\{K_{1,3}\right\}$ ) graphs are hamiltonian. Another conjecture by Thomassen [11] states that all 4-connected line graphs are hamiltonian. As all line graphs are claw-free, the second conjecture appears much weaker than the first, but Ryjáček [10] showed that the two conjectures are actually equivalent.

The general conjecture is still wide open, but several special cases have been solved. In particular, the following result was observed independently by several authors, see, e.g., [2]. The hourglass is the graph on 5 vertices obtained by identifying one vertex of two disjoint triangles.

Theorem 1. Let $G$ be a 4-connected \{claw,hourglass\}-free graph. Then $G$ is hamiltonian.

Very recently, Kaiser et al. showed a similar result for a bigger class of graphs. A graph is said to have the hourglass-property, if every induced hourglass $S$ contains two non-adjacent vertices with a common neighbor outside $S$.

Theorem 2. [7] Every 4-connected claw-free graph with the hourglassproperty is hamiltonian.

Our main result is an extension of Theorem 1 to a slightly different class of graphs. Let $T$ be the line graph of the unique tree $F$ on 8

1991 Mathematics Subject Classification. 05C45.

Key words and phrases. Hamilton cycle, claw-free graphs, forbidden subgraphs. 
vertices with degree sequence $(3,3,3,1,1,1,1,1)$, i.e. $T$ is a chain of three triangles.

Theorem 3. Every 4-connected $\left\{K_{1,3}, T\right\}$-free graph is hamiltonian.

One technical difficulty in the proof of this theorem is that the class of $T$-free graphs is not stable under the closure operation (see Section 3 for a definition). We will actually show a slightly stronger theorem in Theorem 19 to avoid this problem.

In this paper, all graphs are simple. A multigraph may contain multiple edges but no loops. For all terms not defined here we refer the reader to [1]. We denote the neighborhood of a vertex set $X \subseteq V(G)$ in a graph $G$ by $N_{G}(X)$ or $N(X)$, and the closed neighborhood of $X$ is $N_{G}[X]=N[X]=N(X) \cup X$. The degree of a vertex in $v \in V(G)$ is $d_{G}(v)=d(v)=\left|N_{G}(v)\right|$. For $X \subseteq V(G)$, the subgraph induced by $G$ on $X$ is denoted by $G[X]$. We write $L(G)$ for the line graph of $G$. A graph $G$ is essentially $k$-edge-connected if the deletion of less than $k$ edges leaves at most one component with more than one vertex. In this paper by circuit we mean a closed trail, possibly of length zero. A circuit $C$ is dominating if every edge in $G$ is incident to at least one vertex of $C$.

\section{LINE GRAPHS}

In this section, we present some theorems and easy facts about line graphs which we will use in our proofs. An important theorem about hamiltonicity of line graphs is the following classic result by Harary and Nash-Williams.

Theorem 4. [6] If $G$ is a graph and $G=L(H)$ for some multigraph $H$, then $G$ is hamiltonian if and only if $H$ contains a dominating circuit.

Further, the following well known facts will be useful.

Fact 5. If $G$ is a line graph, then there is at most one triangle-free graph $H$ such that $L(H)=G$.

If it exists, we will denote this unique graph by $H=L^{-1}(G)$.

Fact 6. Let $H$ and $H^{\prime}$ be triangle-free graphs. Then $L(H)$ is $L\left(H^{\prime}\right)$-free if and only if $H$ has no subgraph isomorphic to $H^{\prime}$.

Fact 7. For $G=L(H), G$ is $k$-connected if and only if $H$ is essentially $k$-edge-connected. 


\section{Closure And stability}

In a claw-free graph $G$, the vertices can be partitioned into classes, depending on the structure of the graphs $G[N(v)]$ : Let $L C(G)$ denote the class of all vertices for which $G[N(v)]$ is connected, let $L D_{1}(G)$ denote the class of all vertices for which $G[N(v)]$ is disconnected with a component of order one, and let $L D_{2}(G)$ denote the class of all vertices for which $G[N(v)]$ is disconnected with no component of order one. Note that for a vertex $v \in L D_{1}(G) \cup L D_{2}(G), G[N(v)]$ consists of exaxtly two complete graphs.

For a claw-free graph $G$, let $E L(G) \subseteq L C(G)$ denote the class of vertices with a connected but not complete neighborhood. These vertices are called eligible vertices. For an eligible vertex $x \in E L(G)$, let $G^{x}$ denote the graph obtained from $G$ by addition of all missing edges in $G[N(x)]$. Let the closure of $G, \operatorname{cl}(G)$, be the graph obtained from $G$ through repeated application of this local completion, such that $E L(\operatorname{cl}(G))=\emptyset$. This construction was introduced by Ryjáček, and he showed the following statement:

Theorem 8. [10] Let $G$ be a claw-free graph. Then

(i) $\operatorname{cl}(G)$ is unique and well-defined,

(ii) $\operatorname{cl}(G)$ is the line graph of some unique triangle-free graph $H=$ $L^{-1}(\operatorname{cl}(G))$,

(iii) $G$ is hamiltonian if and only if $\operatorname{cl}(G)$ is hamiltonian.

A graph for which $\operatorname{cl}(G)=G$ is called closed.

Lemma 9. Let $G$ be a claw-free graph, and let $v \in E L(G)$. For $i \in$ $\{1,2\}, L D_{i}\left(G^{v}\right) \subseteq L D_{i}(G)$.

Proof. Let $x \in L D_{i}\left(G^{v}\right)$. As $v \in L C\left(G^{v}\right), x \neq v$. If $x v \notin E(G)$, then $N_{G}(x)=N_{G^{v}}(x)$, and, since $E(G) \subseteq E\left(G^{v}\right), x \in L D_{i}(G)$. So suppose that $x v \in E(G)$. Let $N_{1}$ be the component of $G^{v}[N(x)]$ containing $v$, let $N_{2}$ be the other component. As $N(v) \cap N_{2}=\emptyset$, we have $N_{2} \cap N_{G}(x)=$ $N_{2}$. Further, as $v \in E L(G)$, there is another vertex $y \in N_{G}(x) \cap N(v) \subset$ $N_{1}$. Thus, $\left|N_{1} \cap N_{G}(x)\right| \geq 2$ and $\left|N_{2} \cap N_{G}(x)\right|=\left|N_{2}\right|$, showing the lemma.

Corollary 10. For $i \in\{1,2\}, L D_{i}(c l(G)) \subseteq L D_{i}(G)$ for every clawfree graph $G$.

Recently, Broersma and Ryjáček introduced an even stronger concept, the so called cycle closure. Let $G$ be a closed claw-free graph, and let $H=L^{-1}(G)$ be the triangle-free line graph original, i.e. the triangle free graph for which $L(H)=G$. Let $C$ be a cycle of length 
$k$ in $H$, where $4 \leq k \leq 6$ and $C$ contains at least $k-3$ independent vertices of degree 2. Call such a cycle, and its line graph $L(C) \subseteq G$, an eligible cycle. Let the $C$-completion of $G, G_{C}$, be the graph obtained from $G$ by addition of all missing edges in $N[L(C)]$.

Definition 1. We say that a graph $G^{\prime}$ is a cycle closure of $G$, denoted $G^{\prime}=c l_{\text {cyc }}(G)$ if there is a sequence of graphs $G_{1}, G_{2} \ldots, G_{t}$ such that

(i) $G_{1}=\operatorname{cl}(G)$,

(ii) $G_{i+1}=\operatorname{cl}\left(\left(G_{i}\right)_{C}\right)$ for some eligible cycle $C$ in $L^{-1}\left(G_{i}\right), i=$ $1, \ldots, t-1$,

(iii) $G^{\prime}=G_{t}$ contains no eligible cycles.

The following holds for the cycle closure:

Theorem 11. [3] Let $G$ be a claw-free graph. Then

(i) $c l_{c y c}(G)$ is well defined,

(ii) $G$ is hamiltonian if and only if $c l_{c y c}(G)$ is hamiltonian.

We say a graph $G$ is closed under $c l_{\text {cyc }}$ if $\mathrm{cl}_{\text {cyc }}(G)=G$. A similar result as Lemma 9 holds for the cycle closure.

Lemma 12. Let $G$ be a closed claw-free graph, and let $C$ be an eligible cycle in $H=L^{-1}(G)$. For $i \in\{1,2\}, L D_{i}\left(G_{C}\right) \subseteq L D_{i}(G)$.

Proof. Let $C^{\prime}=L(C)$. Let $x \in L D_{i}\left(G_{C}\right)$. As $G_{C}\left[N\left[C^{\prime}\right]\right]$ is complete, $x \notin V\left(C^{\prime}\right)$. If $x \notin N\left(C^{\prime}\right)$, then $N_{G}(x)=N_{G_{C}}(x)$, and, since $E(G) \subseteq$ $E\left(G_{C}\right), x \in L D_{i}(G)$. So suppose that $x \in N\left(C^{\prime}\right)$. Let $N_{1}$ be the component of $G_{C}[N(x)]$ containing $V\left(C^{\prime}\right)$, let $N_{2}$ be the other component. As $N\left(C^{\prime}\right) \cap N_{2}=\emptyset$, we have $N_{2} \cap N_{G}(x)=N_{2}$. Further, as $G$ is clawfree, there are at least two vertices $y, z \in N_{G}(x) \cap V\left(C^{\prime}\right) \subset N_{1}$. Thus, $\left|N_{1} \cap N_{G}(x)\right| \geq 2$ and $\left|N_{2} \cap N_{G}(x)\right|=\left|N_{2}\right|$, showing the lemma.

Corollary 13. For $i \in\{1,2\}, L D_{i}\left(c l_{c y c}(G)\right) \subseteq L D_{i}(G)$ for every clawfree graph $G$.

Let $Y$ be the graph consisting of a central triangle, with an extra triangle attached to each of its three vertices.

We say a class $\mathcal{G}$ of claw-free graphs is stable under the closure (cycle closure) if for every graph $G \in \mathcal{G}$, we have $\mathrm{cl}(G) \in \mathcal{G}\left(\operatorname{cl}_{\text {cyc }}(G) \in \mathcal{G}\right)$. The power of Theorems 8 and 11 lies in the following consequence. If a class $\mathcal{G}$ of claw-free graphs is stable under the closure (cycle closure), and we want to show that all graphs in $\mathcal{G}$ are hamiltonian, then it suffices to consider only the closed graphs in $\mathcal{G}$.

Brousek, Ryjáček and Schiermeyer have characterized all graphs $A$, such that the class of all $\left\{K_{1,3}, A\right\}$-free graphs is stable [4]. Unfortunately, $T$ and $Y$ are not among these graphs. To avoid this difficulty, 

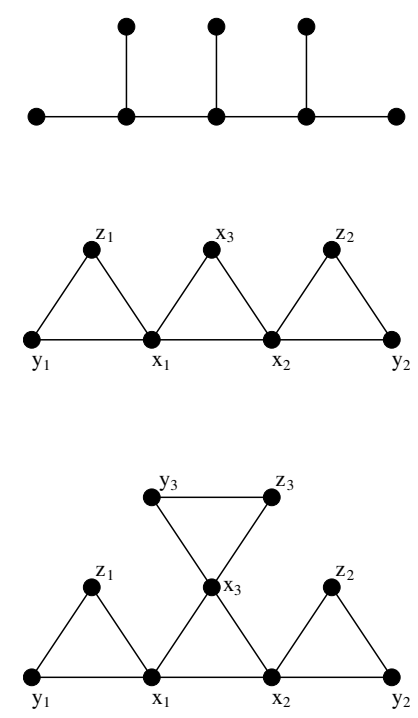

FiguRE 1. F, T and $Y$

we consider a slightly larger class of graphs which is shown to be stable under both closures.

We say that a graph $G$ has the $T$-property ( $Y$-property) if it does not contain an induced copy of $T(Y)$ where the central triangle of $T$ $(Y)$ is the only maximal clique in $G$ containing any of the three edges belonging to the central triangle.

We say that $G$ has the permanent $T$-property ( $Y$-property) if $G$ does not contain a set $X \subseteq V(G)$ such that $G[X]=\operatorname{cl}(G)[X] \cong T$ $(G[X]=\operatorname{cl}(G)[X] \cong Y)$ with the central triangle in $\operatorname{cl}(G)[X]$ being a maximal clique.

Theorem 14. The class of claw-free graphs with the T-property is stable under the closure and under the cycle closure.

Theorem 15. The class of claw-free graphs with the $Y$-property is stable under the closure and under the cycle closure.

Proof of Theorem 14. For the first part of the theorem it suffices to show that $\operatorname{cl}(G)$ has the $T$-property for every claw-free graph $G$ with the permanent $T$-property.

Let $G$ be a claw-free graph with the permanent $T$-property, and let $H=\operatorname{cl}(G)$ be its closure. Suppose, for the sake of contradiction, that $H$ does not have the $T$-property. Let $(G=) G_{0}, G_{1}, \ldots, G_{k}(=H)$ be a sequence of graphs such that $G_{i+1}=G_{i}^{v_{i}}$ for some vertex $v_{i} \in E L\left(G_{i}\right)$ for $0 \leq i \leq k-1$. Let $j$ be maximal such that $G_{j}$ has the permanent 
$T$-property. Without loss of generality we may assume that $j=0$, and let $v=v_{0}$.

There is a vertex set $X=\left\{x_{1}, x_{2}, x_{3}, y_{1}, z_{1}, y_{2}, z_{2}\right\} \subseteq V(G)$ such that $G_{1}[X]$ is a permanent copy of $T$ labeled as in Figure 1 with the triangle $x_{1} x_{2} x_{3}$ being the only maximal clique containing any of the edges $x_{1} x_{2}, x_{1} x_{3}, x_{2} x_{3}$. Since $G$ has the permanent $T$-property and $E(G) \subseteq E\left(G_{1}\right), G[X] \not T$.

Further, $x_{1}, x_{2} \in L D_{2}\left(G_{1}\right)$, thus $x_{1}, x_{2} \in L D_{2}(G)$ by Lemma 9, and therefore $x_{1} x_{2}, x_{1} x_{3}, x_{2} x_{3} \in E(G)$. By symmetry we may assume that one of the edges $x_{1} y_{1}, x_{1} z_{1}, y_{1} z_{1}$ is missing in $G$. If $x_{1} y_{1}, x_{1} z_{1} \in$ $E(G)$ then $G\left[x_{1}, x_{2}, y_{1}, z_{1}\right]$ is a claw, which is not possible. Thus, we may assume by symmetry that $x_{1} y_{1} \notin E(G)$. Since $G_{1}[X] \cong T$, we know that $x_{1}, y_{1} \in N(v)$, in fact there is a shortest $x_{1}-y_{1}$ path $P=x_{1} u_{1} \ldots u_{\ell} y_{1}$ in $N(v)$. Since $G$ has the permanent $T$-property, $H\left[x_{1}, x_{2}, x_{3}, u_{1}, v, y_{2}, z_{2}\right] \not T$, so there must be an edge in $E(H)$ connecting $v$ or $u_{1}$ to $w \in\left\{x_{2}, x_{3}, y_{2}, z_{2}\right\}$. As $x_{1} u_{2} \notin E(G)$ and $x_{1}, u_{2} \in$ $N_{G}(v) \cap N_{G}\left(u_{1}\right)$, we have $v, u_{1} \in L C(G)$, and thus $v, u_{i} \in L C(H)$. As $y_{1} \in N_{H}(v) \cap N_{H}\left(u_{1}\right)$ and $H$ is closed, this implies that $y_{1} w \in E(H)$, a contradiction to the fact that $G_{1}[X]$ is permanent. This shows the first part of the theorem.

For the second part of the theorem, suppose for the sake of contradiction, that there is a closed claw-free graph $G$ with an eligible cycle $C \subseteq G$ such that $G$ has the $T$-property but $G_{C}$ does not have the $T$ property. Hence, there is a vertex set $X=\left\{x_{1}, x_{2}, x_{3}, y_{1}, z_{1}, y_{2}, z_{2}\right\} \subseteq$ $V(G)$ such that $G_{C}[X]$ is a copy of $T$ labeled as in Figure 1 with the triangle $x_{1} x_{2} x_{3}$ being the only maximal clique containing any of the edges $x_{1} x_{2}, x_{1} x_{3}, x_{2} x_{3}$.

Lemma 12 implies that $x_{1} x_{2}, x_{1} x_{3}, x_{2} x_{3} \in E(G)$, and we may assume by symmetry (and $G$ being claw-free) that $x_{1} y_{1} \notin E(G)$, following the same argument as in the first part of the proof. Thus, $y_{1} \in N_{G}[C]$ and $x_{1} \in N_{G}(C)$. Since $G$ is claw-free and $C$ is an induced cycle, there are two consecutive vertices $y, z \in V(C) \cap N_{G}\left(x_{1}\right)$. By the construction of $G_{C}, N_{G_{C}}[y]=N_{G_{C}}[z] \subseteq N_{G_{C}}\left[y_{1}\right]$, and therefore, $G\left[x_{1}, x_{2}, x_{3}, y, z, y_{2}, z_{2}\right] \cong T$, contradicting the fact that $G$ has the $T$-property.

Proof of Theorem 15. The proof is almost identical to the proof of Theorem 14, the details are left to the reader. 


\section{Collapsible graphs}

Catlin [5] introduced the concept of collapsible graphs. A multigraph $G$ is collapsible if for any even subset $X \subset V(G)$, there is a submultigraph $R$ such that $G-E(R)$ is connected and $X$ is precisely the set of odd degree vertices of $R$. If $H$ is a connected submultigraph of $G$, then the multigraph $G / H$ is obtained from $G$ by contracting $H$ to a single vertex, discarding all loops. These are a few very useful results using the concept.

Theorem 16. [5] Let $H$ be a collapsible submultigraph of $G$. Then $G$ has a spanning closed trail if and only if $G / H$ does.

Theorem 17. [5] Any 4-edge-connected multigraph is collapsible.

Lai proved the following result.

Theorem 18. [8] Let $G$ be a 2-connected multigraph with minimum degree $\delta(G) \geq 3$. If every edge of $G$ is contained in a cycle of length at most 4 , then $G$ is collapsible.

\section{Hamiltonicity}

In this section we show the following theorem which implies Theorem 3.

Theorem 19. Every 4-connected claw-free graph with the T-property is hamiltonian.

Consider the following operation on a claw-free graph $G$ closed under $\mathrm{cl}_{\text {cyc }}$. Let $H$ be the triangle-free graph such that $G=L(H)$. Let $H_{e}$ be the graph obtained from $H$ by subdividing an edge $e$ whose endvertices both have degree greater than 3 . Let $G_{e}=L\left(H_{e}\right)$.

Proposition 20. Let $G$ be a claw-free graph closed under $c_{c y c}$, let $H=L^{-1}(G)$ and $e \in E(H)$ be an edge with endvertices of degree greater than 3. Then:

(i) $G_{e}$ is closed under $c l_{c y c}$.

(ii) If $G$ is 4-connected, then $G_{e}$ is 4-connected.

(iii) If $G_{e}$ is hamiltonian, then $G$ is hamiltonian.

Proof. As a line graph of a triangle free graph, $G_{e}$ is closed. Further, since $H$ is triangle-free without eligible cycles, $H_{e}$ contains no eligible cycles, so $G_{e}$ is closed under $\mathrm{cl}_{\text {cyc }}$.

Let $G$ be 4 -connected. This is equivalent to the statement that $H$ is essentially 4-edge-connected. Every essential edge-cut in $H_{e}$ induces an edge-cut of at most the same size in $H$. This induced cut is essential, 
unless it contains $e$. But there is no edge cut in $H$ of size smaller than 4 containing the edge $e$ since its endvertices both have degree greater than three. Thus, $H_{e}$ is essentially 4-edge-connected. This implies that $G_{e}$ is 4-connected, showing (ii).

Now let $G_{e}$ be hamiltonian with a hamiltonian cycle $C$. The preimage $L^{-1}(C)$ is a dominating circuit in $H_{e}$. Clearly, reversing the subdivision of $e$ in $H_{e}$ yields a dominating circuit in $H$, and thus a hamiltonian cycle in $G$, showing (iii).

Let $G$ be a claw-free graph closed under $\mathrm{cl}_{\text {cyc }}$ with the $T$-property. Repeat the above operation until the resulting graph $G^{\prime}=s(G)$ has the following properties:

(i) $G^{\prime}$ has the $T$-property,

(ii) For every edge $e \in L^{-1}\left(G^{\prime}\right)$ with both endvertices having degree greater than $3, G_{e}^{\prime}$ does not have the $T$-property.

The graph $s(G)$ may not be unique, but this does not affect the validity of the argument.

To show Theorem 19 it suffices to show it for such a graph $s\left(\operatorname{cl}_{\mathrm{cyc}}(G)\right)$ by Theorem 11 and Proposition 20. To simplify notation we may assume that $G=s\left(\mathrm{cl}_{\text {cyc }}(G)\right)$. Let $H=L^{-1}(G)$, and we want to show that $H$ contains a dominating circuit. Let $A=\{v \in V(H) \mid d(v)=1\}$, $B=\{v \in V(H) \mid d(v)=2\}$.

Let $K$ be a component of $H-(A \cup B)$. We want to show that $K$ is collapsible. If $K$ contains only one vertex, there is nothing to show, so we may assume that $K$ contains at least two vertices. Let $R$ be a block of $K$. The proof is structured as a series of claims.

Claim 1. $\delta(R) \geq 2$, i.e. $R \nsubseteq K_{2}$.

Let $x \in V(R)$, and suppose that $d_{R}(x)=1$. If $d_{H}(x)=3$, let $N(x)=\left\{x_{1}, x_{2}, x_{3}\right\}$, with $x_{1} \in V(R)$. Since $H$ is essentially 4-edgeconnected, $d_{H}\left(x_{i}\right) \geq 3$. Let $y_{i}, z_{i} \in N\left(x_{i}\right)$ for $i \in\{1,2\}$. As $H$ is triangle-free and contains no eligible cycles, $H\left[x, x_{1}, y_{1}, z_{1}, x_{2}, y_{2}, z_{2}, x_{3}\right]$ contains a copy of $F$ with $x$ as central vertex, a contradiction. Thus, $d_{H}(x) \geq 4$.

Let $x_{1} \in N(x) \cap V(R)$. By the same argument, $d_{H}\left(x_{1}\right) \geq 4$. Let $s$ be the new vertex in $H_{x x_{1}}$. Then there is a vertex $v$ of degree 3 , and vertices $w, y, z, y_{1} \in V(H)$ such that $H_{x x_{1}}\left[v, x_{1}, y_{1}, s, y, x, z, w\right]$ contains $F$ with center vertex $v$. But then $H\left[x, x_{1}\right]$ is not a block of $H-(A \cup B)$, the contradiction establishing the claim.

Claim 2. Let $x, v \in V(R)$ with $d_{R}(x)=2$ and $d_{H}(v)=3$. Then $x v \notin E(H)$. 
If $d_{H}(x)=3$, let $x_{1} \in N(x) \backslash V(R)$. By Claim 1, $x_{1} \notin V(H) \backslash(A \cup$ $B)$, thus $d_{H}\left(x_{1}\right) \leq 2$, a contradiction to $H$ being essentially 4-edgeconnected . Therefore, $d_{H}(x) \geq 4$.

Let $x_{1}, x_{2} \in N(x) \backslash V(R)$, let $y, z \in N(v) \backslash\{x\}$, and let $y_{1}, y_{2} \in$ $N(y) \backslash\{v\}$. If $x v \in E(H)$, then $H\left[v, x, x_{1}, x_{2}, y, y_{1}, y_{2}, z\right]$ contains $F$ with center vertex $v$, a contradiction establishing the claim.

Let $X=\left\{x \in V(R) \mid d_{R}(x)=2\right\}$.

Claim 3. For any $x \in X, d_{H}(x) \geq 4$.

Suppose for the sake of contradiction that $d_{H}(x)=3$, and let $y \in$ $N(x) \backslash V(R)$. If $y \in A \cup B$, then an essential edge cut of size at most three exists in $H$, so this is not the case. Thus, $y \in V(K) \backslash V(R)$. But this implies that $x y$ is a block of $K$ contrary to Claim 1.

Claim 4. For any pair $x_{1}, x_{2} \in X, N\left[x_{1}\right] \cap N\left(x_{2}\right) \cap V(R)=\emptyset$.

Suppose first that $x_{1} x_{2} \in E(H)$. Then, as $G_{x_{1} x_{2}}$ does not have the $T$-property, there is a path of length 3 in $R$ from $x_{1}$ to $x_{2}$ with one of the two internal vertices having degree 3 in $H$. But this is impossible by Claim 2 .

Now suppose that there is a vertex $y \in N\left(x_{1}\right) \cap N\left(x_{2}\right) \cap V(R)$. As $G_{x_{1} y}$ does not have the $T$-property, there is a path $x_{1} y_{1} v y$ in $R$ with $d_{H}(v)=3$ and, by Claim $2, d_{H}(y), d_{H}\left(y_{1}\right) \geq 4$. Let $y_{2} \in N(v) \backslash\left\{y_{1}, y\right\}$, and $x_{3} \in N(y) \backslash\left\{v, x_{1}, x_{2}\right\}$. Then $N\left(y_{1}\right)=\left\{v, x_{1}, x_{2}, x_{3}\right\}$, since otherwise a copy of $F$ with $v$ as center can be found. Similarly, $N\left(y_{2}\right) \subseteq$ $\left\{v, x_{1}, x_{2}, x_{3}\right\}$. By Claim $2, d_{R}\left(y_{2}\right) \geq 3$, and so $y_{2} \in N\left(x_{1}\right) \cup N\left(x_{2}\right)$, which in turn guarantees that $d_{H}\left(y_{2}\right) \geq 4$ and therefore $N\left(y_{2}\right)=$ $\left\{v, x_{1}, x_{2}, x_{3}\right\}$. But this implies that $d_{R}\left(x_{1}\right), d_{R}\left(x_{2}\right) \geq 3$, a contradiction.

Claim 5. $\delta(R-X)=3$.

Since $K$ is not trivial and $|N(v) \cap X| \leq 1$ for every $v \in V(R) \backslash X$ by Claim $4, \delta(R-X) \geq 2$. If $\delta(R-X) \geq 4$, then $G_{e}$ has the $T$-property for every $e \in E(R-X)$, a contradiction, so $2 \leq \delta(R-X) \geq 3$.

Suppose for the sake of contradiction that $\delta(R-X)=2$. Let $y_{1} \in$ $V(R) \backslash X$ with $d_{R-X}\left(y_{1}\right)=2$, and let $x \in X$ be its only neighbor in $X$. Let $y_{2} \in V(R) \backslash X$ be the other neighbor of $x$ in $R$. Since $G_{x y_{1}}$ does not have the $T$-property, there is a vertex $v \in N\left(y_{1}\right) \cap N\left(y_{2}\right)$ with $d_{H}(v)=3$. By Claim 2, there are vertices $z_{1}, z_{2} \in N\left(y_{1}\right) \backslash\{x, v\}$. Now, $N\left(y_{1}\right)=N\left(y_{2}\right)=\left\{x, v, z_{1}, z_{2}\right\}$, otherwise a copy of $F$ with center $v$ exists. Further, $z_{1}, z_{2} \notin B$ as there are no eligible cycles in $H$. Finally, 
$z_{1}, z_{2} \notin X$ by Claim 4 . Thus $d_{R-X}\left(y_{1}\right)=d_{R-X}\left(y_{2}\right)=3$, a contradiction establishing the claim.

Claim 6. Every edge e $\in E(R-X)$ lies on a $C_{4} \subset R-X$.

Let $e=x y$. If $d_{H}(x), d_{H}(y) \geq 4$, there exists a path $x v w y$ through $R$ with $d_{H}(v)=3$ or $d_{H}(w)=3$ since $G_{x y}$ does not have the $T$ property. Then, $v, w \notin X$ by Claim 2 , so $x v w y x$ is the desired $C_{4}$. Thus, suppose that $d_{H}(x)=3$. Let $N(x)=\{u, v, y\} \subseteq V(R) \backslash X$. Then $|(N(v) \cup N(y)) \backslash\{x\}| \leq 3$, otherwise a copy of $F$ with $x$ as center can be found. Together with Claim 5, this implies the existence of the $C_{4}$.

Claim 7. $R-X$ is 2-connected.

Suppose the claim is false. Since $R$ is 2-connected and $X$ is an independent set by Claim 4 , there is a vertex $x \in X$ with neighbors $y_{1}$ and $y_{2}$ which are not in the same block of $R-X$. But the same argument as in the proof of Claim 5 shows that $N\left(y_{1}\right)=N\left(y_{2}\right)$, a contradiction.

Claim 8. $R-X$ is collapsible.

This follows directly from Theorem 18 with Claims 5, 6 and 7 . $\diamond$

To show Theorem 19, start with $H-(A \cup B)$ and, for every vertex in $B$, add an edge between its two neighbors. Call the resulting graph $H^{\prime}$. Contract $R-X$ in $H^{\prime}$ for each block $R$ of each component of $H-(A \cup$ $B$ ). The resulting multigraph $H^{\prime \prime}$ is essentially 4-edge-connected with minimum degree at least 4, and thus 4-edge-connected. By Theorem 17, $H^{\prime \prime}$ is collapsible, and thus $H^{\prime}$ has a spanning circuit by Theorem 16 . All vertices in $A \cup B$ are independent, and so $H$ has a dominating circuit, and therefore $G=L(H)$ is hamiltonian.

\section{Closing Remarks}

The techniques used in the proof of Theorem 19 are not quite sufficent to prove a similar result about hamiltonicity of 4-connected clawfree graphs with the weaker $Y$-property. Take for example the graph $H$ in Figure 6, and let $G=L(H)$. Clearly, $G$ is 4-connected, claw-free, cycle closed and has the $Y$-property. But if we define $A, B$ and $X$ as above, $H-(A \cup B \cup X)$ consists of two non-collapsible $C_{4}$ s.

Similarly, one can define the weak hourglass-property as follows. A graph $G$ is said to have the weak hourglass-property, if for every set $S \subseteq V(G)$ with $G$ inducing an hourglass on $S$, one of the following is true: 


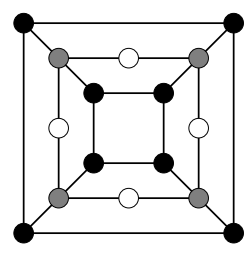

Figure 2. A line graph original of a graph with the $Y$-property

(i) There is a common neighbor outside of $S$ for two of the nonadjacent vertices in $S$, or

(ii) both edges in $G[S]$ not incident to the center vertex in $S$ lie in cliques which are not subsets of $S$.

This property implies the $T$-property and the hourglass-property. It is easy to see that the class of graphs with the weak hourglass-property is stable under both the closure and the cycle closure, but again, the proof techniques used in the proof of Theorem 19 are not quite sufficent to prove a similar result about hamiltonicity of 4-connected claw-free graphs with this property. The graph pictured in Figure 3 could be a block $R$ of $H-(A \cup B)$, but $R-X$ contains vertices of degree 1 , and is thus not collapsible.

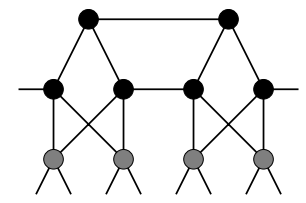

Figure 3. A non-collapsible block

\section{REFERENCES}

1. B. Bollobás, "Modern Graph Theory," Springer Verlag, New York, 1998.

2. H. Broersma, M. Kriesell, Z. Ryjáček, On factors of 4-connected claw-free graphs, J. Graph Theory 37 (2001), 125-136.

3. H. Broersma, Z. Ryjáček, Strengthening the closure concept in claw-free graphs, Discrete Math. 233 (2001), no. 1-3, 55-63.

4. J. Brousek, Z. Ryjáček, I. Schiermeyer, Forbidden subgraphs, stability and hamiltonicity, Discrete Math. 197/198 (1999), 143-155.

5. P. Catlin, A reduction method to find spanning eulerian subgraphs, J. Graph Theory 12 (1988), 29-45.

6. F. Harary, C. St. J. A. Nash-Williams, On eulerian and hamiltonian graphs and line graphs, Can. Math. Bull 8 (1965) 701-710.

7. T. Kaiser, M. Li, Z. Ryjáček, L. Xiong, Hourglasses and Hamilton cycles in 4-connected claw-free graphs, preprint. 
8. H. J. Lai, Graphs whoseedges are in small cycles, Discrete Math. 94 (1991), $11-22$.

9. H. M. Matthews, D. P. Sumner, Hamiltonian results in $K_{1,3}$-free graphs, J. Graph Theory 8 (1984), 139-146.

10. Z. Ryjáček, On a closure concept in claw-free graphs, J. Combin. Theory, Ser. B 70 (1997), 217-224.

11. C. Thomassen, Reflections on graph theory, J. Graph Theory 10 (1986), 309324 .

Technische Universität Berlin, Fachbereich Mathematk, MA 6-2, Strasse des 17. Juni 136, 10623 Berlin, Germany

E-mail address: <fpfender@math.tu-berlin.de> 